\title{
Control and detection of singlet-triplet mixing in a random nuclear field
}

\author{
F. H. L. Koppens, J. A. Folk, J. M. Elzerman, R. Hanson, L. H. Willems \\ van Beveren, I. T. Vink, L. P. Kouwenhoven, and L. M. K. Vandersypen \\ Kavli Institute of NanoScience Delft, \\ P.O. Box 5046, 2600 GA Delft, The Netherlands \\ H. P. Tranitz and W. Wegscheider \\ Institut für Angewandte und Experimentelle Physik, \\ Universität Regensburg, Regensburg, Germany
}

\begin{abstract}
We observe mixing between two-electron singlet and triplet states in a double quantum dot, caused by interactions with nuclear spins in the host semiconductor. This mixing is suppressed by applying a small magnetic field, or by increasing the interdot tunnel coupling and thereby the singlet-triplet splitting. Electron transport involving transitions between triplets and singlets in turn polarizes the nuclei, resulting in striking bistabilities. We extract from the fluctuating nuclear field a limitation on the time-averaged spin coherence time $T_{2}^{*}$ of $25 \mathrm{~ns}$. Control of the electronnuclear interaction will therefore be crucial for the coherent manipulation of individual electron spins.
\end{abstract}


A single electron confined in a GaAs quantum dot is often referred to as artificial hydrogen. One important difference between natural and artificial hydrogen, however, is that in the first, the hyperfine interaction couples the electron to a single nucleus, while in artificial hydrogen the electron is coupled to about a million Ga and As nuclei. This creates a subtle interplay between electron spin eigenstates affected by the ensemble of nuclear spins (the Overhauser shift), nuclear spin states affected by time-averaged electron polarization (the Knight shift), and the flip-flop mechanism that trades electron and nuclear spins [1, 2].

The electron-nuclear interaction has important consequences for quantum information processing with confined electron spins [3]. Any randomness in the Overhauser shift introduces errors in a qubit state, if no correcting measures are taken [4, 5, 6]. Even worse, multiple qubit states, like the entangled states of two coupled electron spins, are redefined by different Overhauser fields. Characterization and control of this mechanism will be critical both for identifying the problems as well as finding potential solutions.

Here, the implications of the hyperfine interaction on entangled spin states are studied in two coupled quantum dots — an artificial hydrogen molecule — where the molecular states can be controlled electrically. A random polarization of nuclear spins creates an inhomogeneous effective field that couples molecular singlet and triplet states, and leads to new eigenstates that are admixtures of these two. We use transport measurements to determine the degree of mixing over a wide range of tunnel coupling, and observe a subtle dependence of this mixing on magnetic field. We find that we can controllably suppress the mixing by increasing the singlet-triplet splitting. This ability is crucial for reliable two-qubit operations such as the SWAP gate [3].

Furthermore, we find that electron transport itself acts back on the nuclear spins through the hyperfine interaction, and time-domain measurements reveal complex, often bistable, behavior of the nuclear polarization. Understanding the current-induced nuclear polarization is an important step towards electrical control of the nuclear spins. Such control will be critical for electrical generation and detection of entangled nuclear spin states [7], or for transfer of quantum information between electron and nuclear spin systems [8, 9]. Even more appealing will be reducing the nuclear field fluctuations in order to achieve longer electron spin coherence times [10, 11, 12].

The coupled electron-nuclear system is studied using electrical transport measurements through two dots in series [13], in a regime where the Pauli exclusion principle blocks current 
flow [14, 15]. The dots are defined using electrostatic gates on a GaAs/AlGaAs heterostructure (see Fig. 1E and [16]). The gate voltages are tuned such that one electron always resides in the right dot, and current flows if a second electron tunnels from the left reservoir, through the left and right dots, to the right reservoir (see Fig. 1D). This cycle can be described using the occupations $(m, n)$ of the left and right dots: $(0,1) \rightarrow(1,1) \rightarrow(0,2) \rightarrow(0,1)$. When an electron enters from the left dot, the two-electron system forms either a molecular singlet, $\mathrm{S}(1,1)$, or a molecular triplet, $\mathrm{T}(1,1)$. From $\mathrm{S}(1,1)$, the electron in the left dot can move to the right dot to form $\mathrm{S}(0,2)$. From $\mathrm{T}(1,1)$, however, the transition to $(0,2)$ is forbidden by spin conservation $(\mathrm{T}(0,2)$ is much higher in energy than $\mathrm{S}(0,2))$. Thus, as soon as $\mathrm{T}(1,1)$ is occupied, further current flow is blocked (Pauli blockade).

A characteristic measurement of this blockade is shown in Fig. 1A. The suppression of current $(<80 \mathrm{fA})$ in the region defined by dashed lines is a signature of Pauli blockade [14, 15] (see also Fig. S1 and [17]). Fig. 1B shows a similar measurement, but with a much weaker interdot tunnel coupling $t$. Strikingly, a large leakage current now appears in the Pauli blockaded region, even though the barrier between the two dots is more opaque. Furthermore, this leakage current is substantially reduced by an external magnetic field of only $100 \mathrm{mT}$ (Fig. 1C). Such a strong field dependence is remarkable because the in-plane magnetic field, $B_{\text {ext }}$, couples primarily to spin and the Zeeman energies involved are very small $\left(E_{Z} \sim 2.5 \mu \mathrm{eV}\right.$ at $B_{\text {ext }}=100 \mathrm{mT}$ compared to the thermal energy, $\sim 15 \mu \mathrm{eV}$ at 150 $\mathrm{mK}$, for example).

Leakage in the Pauli blockade regime occurs when singlet and triplet states are coupled. The $\mathrm{T}(1,1)$ state that would block current can then transition to the $\mathrm{S}(1,1)$ state and the blockade is lifted (Fig. 1D). As we will show, coupling of singlets and triplets in Figs. 1B,C originates from the hyperfine interaction between the electron spins and the Ga and As nuclear spins (other leakage mechanisms can be ruled out, see [17]).

The hyperfine interaction between an electron with spin $\vec{S}$ and a nucleus with spin $\vec{I}$ has the form $A \vec{I} \cdot \vec{S}$, where $A$ characterizes the coupling strength. An electron coupled to an ensemble of $n$ nuclear spins experiences an effective magnetic field $\overrightarrow{B_{N}} \sim \frac{1}{g \mu_{B}} \sum_{i}^{n} A_{i} \overrightarrow{I_{i}}$, with $g$ the electron $g$-factor and $\mu_{B}$ the Bohr magneton [1]. For fully polarized nuclear spins in GaAs, $B_{N} \sim 5 \mathrm{~T}$ [18]. For unpolarized nuclear spins, statistical fluctuations give rise to an effective field pointing in a random direction with an average magnitude of $5 \mathrm{~T} / \sqrt{n}$ 44, 5, 19]. Quantum dots like those measured here contain $n \sim 10^{6}$ nuclei, so $\left\|\overrightarrow{B_{N}}\right\| \sim 5 \mathrm{mT}$. 
Nuclei in two different dots give rise to effective nuclear fields, $\overrightarrow{B_{N 1}}$ and $\overrightarrow{B_{N 2}}$, that are uncorrelated. Although the difference in field $\overrightarrow{\Delta B_{N}}=\overrightarrow{B_{N 1}}-\overrightarrow{B_{N 2}}$ is small, corresponding to an energy $E_{N} \equiv g \mu_{B}\left\|\Delta \overrightarrow{B_{N}}\right\| \sim 0.1 \mu \mathrm{eV}$, it nevertheless plays a critical role in Pauli blockade. The $(1,1)$ triplet state that blocks current flow consists of one electron on each of the two dots. When these two electrons are subject to different fields, the triplet is mixed with the singlet and Pauli blockade is lifted. For instance, an inhomogeneous field along $\hat{z}$ causes the triplet $\left|T_{0}\right\rangle=\frac{1}{\sqrt{2}}(|\uparrow \downarrow\rangle+|\downarrow \uparrow\rangle)$ to evolve into the singlet $\frac{1}{\sqrt{2}}(|\uparrow \downarrow\rangle-|\downarrow \uparrow\rangle)$. Similarly, the other two triplet states, $\left|T_{+}\right\rangle=|\uparrow \uparrow\rangle$ and $\left|T_{-}\right\rangle=|\downarrow \downarrow\rangle$, evolve into the singlet due to $\hat{x}$ and $\hat{y}$ components of $\overrightarrow{\Delta B_{N}}$.

The degree of mixing by the inhomogeneous field depends on the singlet-triplet energy splitting, $E_{S T}$. Singlet and triplet states that are close together in energy $\left(E_{S T} \ll E_{N}\right)$ are strongly mixed, while states far apart in energy $\left(E_{S T} \gg E_{N}\right)$ experience only a slight perturbation due to the nuclei.

The singlet-triplet splitting depends on the interdot tunnel coupling $t$ and on the detuning of left and right dot potentials $\Delta_{L R} . \Delta_{L R}$ and $t$ are controlled experimentally using gate voltages (Fig. 1E). $V_{t}$ controls the interdot tunnel coupling. $V_{L}$ and $V_{R}$ set the detuning, and thereby determine whether transport is inelastic (detuned levels), resonant (aligned levels), or blocked by Coulomb blockade (Fig. 1F). The coupling of the dots to the leads is held constant using $V_{\text {lead }}$.

The effect of the two tunable parameters $t$ and $\Delta_{L R}$ on the singlet and triplet energies is illustrated in Figs. 2A and 2B. For weak tunnel coupling $(t \sim 0)$, and in the absence of a hyperfine interaction $\left(E_{N} \sim 0\right)$, the $(1,1)$ singlet and $(1,1)$ triplet states are nearly degenerate (Fig. 2A). A finite interdot tunnel coupling $t$ leads to an anticrossing of $\mathrm{S}(1,1)$ and $\mathrm{S}(0,2)$. The level repulsion results in an increased singlet-triplet splitting that is strongly dependent on detuning (Fig. 2B). At the resonant condition $\left(\Delta_{L R}=0\right.$, aligned levels), the two new singlet eigenstates are equidistant from the triplet state, both with $E_{S T}=\sqrt{2} t$. For finite detuning (but smaller than the single dot S-T splitting), one singlet state comes closer to the triplet state $\left(E_{S T} \sim t^{2} / \Delta_{L R}\right)$, while the other moves away. Both in Figs. $2 \mathrm{~A}$ and $2 \mathrm{~B}$, singlet and triplet states are pure eigenstates (not mixed) and therefore Pauli blockade would be complete.

The additional effect of the inhomogeneous nuclear field is shown in Figs. 2C and 2D. For small $t\left(\sqrt{2} t, t^{2} / \Delta_{L R}<E_{N}\right)$, the $(1,1)$ singlet and $(1,1)$ triplet are close together in energy 
and hence mix strongly (purple lines) over the entire range of detuning. For $t$ such that $t^{2} / \Delta_{L R}<E_{N}<\sqrt{2} t$, triplet and singlet states mix strongly only for finite detuning. This is because $E_{S T}$ is larger than $E_{N}$ for aligned levels but smaller than $E_{N}$ at finite detuning. For still larger $t\left(\sqrt{2} t, t^{2} / \Delta_{L R}>E_{N}\right.$, not shown in Fig. 2), mixing is weak over the entire range of detuning. In the cases where mixing between $\mathrm{S}$ and $\mathrm{T}$ is strong, as in Figs. $2 \mathrm{C}$ and D (for large detuning), Pauli blockade is lifted and a leakage current results.

The competition between $E_{S T}$ and $E_{N}$ can be seen experimentally by comparing 1D traces of leakage current as a function of detuning over a wide range of $t$ (Fig. 3A). Resonant current appears as a peak at $\Delta_{L R}=0$; inelastic leakage as the shoulder at $\Delta_{L R}>0$ [20]. When the interdot tunnel coupling is small, both resonant and inelastic transport are allowed due to singlet-triplet mixing, and both rise as the middle barrier becomes more transparent. As the tunnel coupling is raised further, the point is reached where $E_{S T}$ becomes larger than the nuclear field and Pauli blockade suppresses the current (see also Fig. 1A). The maximum resonant current occurs at a smaller value of $t$ compared to the maximum inelastic current (see inset in Fig. 3A). This is consistent with $E_{S T}$ being much smaller for finite detuning than for aligned levels, $t^{2} / \Delta_{L R} \ll \sqrt{2} t$ (Figs. 2B, D).

The experimental knob provided by electrostatic gates is very coarse on the energy scales relevant to the hyperfine interaction. However, the external magnetic field can easily be controlled with a precision of $0.1 \mathrm{mT}$, corresponding to a Zeeman splitting (2 neV) that is fifty times smaller than $E_{N}$. For this reason, monitoring the field dependence allows a more detailed examination of the competing energy scales $E_{S T}, E_{Z}$ and $E_{N}$.

The competition between $E_{Z}$ and $E_{N}$ is clear for small interdot tunnel coupling (Fig. 3B). Leakage current is suppressed monotonically with magnetic field, on a scale of $\sim 5 \mathrm{mT}$ and $\sim 10 \mathrm{mT}$ for inelastic and resonant transport respectively. The qualitative features of this field dependence can be understood from the insets in Fig. 2C. At zero field all states are mixed strongly by the inhomogeneous nuclear field, but when $E_{Z}$ exceeds $E_{N}$, the mixing between the singlet and two of the triplet states $\left(\left|T_{+}\right\rangle\right.$and $\left.\left|T_{-}\right\rangle\right)$is suppressed. An electron loaded into either of these blocks further current flow and leakage disappears.

The magnitude of the fluctuating Overhauser field can be extracted from the inelastic peak shape in the limit of small $t$ (as in the inset of Fig. 3B). A fit of the data with a model that describes the transport cycle using the density matrix approach [21] is presented in [17]. From this fit, we find a magnitude of the inhomogeneous field $\sqrt{\left\langle\Delta B_{N}{ }^{2}\right\rangle}=1.73 \pm 0.02 \mathrm{mT}$ 
$\left(E_{N}=0.04 \mu \mathrm{eV}\right)$, largely independent of $\Delta_{L R}$ over the parameter range studied [22]. The value for the effective nuclear field fluctuations in a single dot is obtained from the relation $\left\langle B_{N}{ }^{2}\right\rangle=\frac{1}{2}\left\langle\Delta B_{N}{ }^{2}\right\rangle$, giving $\sqrt{\left\langle B_{N}{ }^{2}\right\rangle}=1.22 \mathrm{mT}$. This is consistent with the strength of the hyperfine interaction in GaAs and the number of nuclei that are expected in each dot [4, 23].

The three-way interplay between $E_{S T}, E_{Z}$ and $E_{N}$ is most clearly visible in the resonant current. At an intermediate value of tunnel coupling, $t \gtrsim E_{N}$ (Fig. 3C), the resonant peak is split in magnetic field, with maxima at $\pm 10 \mathrm{mT}$ (see inset). This behavior can be understood from the lower inset in Fig. 2D. At $B_{\text {ext }}=0$, the current is somewhat suppressed compared to Fig. 3B, because now $E_{S T}>E_{N}$. Increasing $B_{\text {ext }}$ enhances the mixing as the $\left|T_{+}\right\rangle$and $\left|T_{-}\right\rangle$states approach the singlet states. The maximum leakage occurs when the states cross, at $E_{S T}(=\sqrt{2} t)=E_{Z}$. Here, $E_{Z}=0.25 \pm 0.03 \mu \mathrm{eV}$ at the current maximum, from which we can extract $t=0.18 \pm 0.02 \mu \mathrm{eV}$ for this setting of $V_{t}$. At still larger $B_{\text {ext }},\left|T_{+}\right\rangle$and $\left|T_{-}\right\rangle$ move away from the singlet states again, and the leakage current is suppressed.

The system enters into a new regime for still higher tunnel coupling (Figs.3D and 4), where it becomes clear that the electron-nuclear system is dynamic. The zero field resonant leakage is further suppressed and above $10 \mathrm{mT}$ prominent current spikes appear (left inset). The spikes are more dramatically visible in a 3D surface plot of leakage over a broader range of field (Fig. 4A). Even for fixed experimental parameters, the current fluctuates in time as shown in Fig. 4B.

We find that time dependent behavior is a consistent feature of resonant transport for $\left(E_{S T}, E_{Z}\right) \gg E_{N}$. For some settings the time dependence is fast (see, e.g., the fluctuations of Figs. $4 \mathrm{~A}$ and 4B), but for others the leakage changes much more slowly. An example of the slower time dependence is shown in Fig. 4C. Starting from an equilibrium situtation (bias voltage switched off for five minutes), the current is initially very small after the bias is turned on. It builds up and then saturates after a time that ranges from less than a second to several minutes. This timescale depends on $\Delta_{L R}, t$, and $B_{\text {ext }}$. When no voltage bias is applied, the system returns to equilibrium after $\sim 80$ s at $200 \mathrm{mT}$. Similar long timescales of the nuclear spin-lattice relaxation times have been reported before in GaAs systems [24] and quantum dots [25]. We thus associate these effects with current-induced dynamic nuclear polarization and relaxation.

Evidence that the fast fluctuations too are related to current induced nuclear polarization (and cannot be explained by fluctuating background charges alone), is found in their depen- 
dence on sweep direction and sweep rate [24, 26]. When the magnetic field is swept while maintaining fixed $\Delta_{L R}$, the current shows fluctuations at low field but suddenly becomes stable at high field (Fig. 4D). The crossover from unstable to stable behavior occurs at a field that is hysteretic in sweep direction (Fig. 4D), and this hysteresis becomes more pronounced at higher sweep rates (faster than $\sim 1 \mathrm{mT} / \mathrm{s}$ ). The connection between the fluctuations and nuclear polarization is also evident from time traces, where instability develops only after the nuclear polarization is allowed to build for some time (Fig. S3).

Unlike the regular oscillations that have been observed in other GaAs structures (see e.g. 1, 27]), the fluctuations in our measurements are random in time, and in many cases suggest bistability with leakage current moving between two stable values. We discuss the origin of such fast bistable fluctuations in [17].

The ensemble of random nuclear spins that gives rise to the mixing of two-electron states as observed in this experiment also gives rise to an uncertainty of $g \mu_{B} \sqrt{\left\langle B_{N}{ }^{2}\right\rangle}=0.03 \mu \mathrm{eV}$ in the Zeeman energy of one electron. When averaged over a time longer than the correlation time of the nuclear spin bath $(\sim 100 \mu \mathrm{s})$ [28], this implies an upper limit on the dephasing time of $T_{2}^{*}=\hbar / g \mu_{B} \sqrt{\frac{2}{3}\left\langle B_{N}^{2}\right\rangle}=25 \mathrm{~ns}$ (following the definition of [4]), comparable to the $T_{2}^{*}$ found in recent optical spectroscopy measurements [29]. This value is four orders of magnitude shorter than the theoretical prediction for the electron spin $T_{2}$ in the absence of nuclei, which is limited only by spin-orbit interactions [30, 31, 32].

One way to eliminate the uncertainty in Zeeman energy that leads to effective dephasing is to maintain a well-defined nuclear spin polarization [12]. Many of the regimes explored in this paper show leakage current that is stable when current-induced polarization is allowed to settle for some time. These may in fact be examples of specific nuclear polarizations that are maintained electrically.

\section{Supporting online text and figures}

(www.sciencemag.org/cgi/content/full/1113719/DC1) 
[1] Optical Orientation, edited by F. Meier and B.P. Zakharchenya (North-Holland, New York, 1984).

[2] D. Gammon et al., Science 277, 85 (1997).

[3] D. Loss, D.P. DiVincenzo, Phys. Rev. A 57, 120 (1998).

[4] I.A. Merkulov, A.L. Efros, J. Rosen, Phys. Rev. B 65, 205309 (2002).

[5] P.-F. Braun et al., Phys. Rev. Lett. 94, 116601 (2005).

[6] A.S. Bracker et al., Phys. Rev. Lett. 94, 047402 (2005).

[7] M. Eto, T. Ashiwa, M. Murata, J. Phys. Soc. Jpn. 73, 307 (2004).

[8] B.E. Kane, Nature 393, 133 (1998).

[9] J. M. Taylor, A. Imamoglu, M. D. Lukin, Phys. Rev. Lett. 91, 246802 (2003).

[10] J. Schliemann, A. Khaetskii, D. Loss, J. Phys. Condens. Matter 15, R1809-R1833 (2003).

[11] A. V. Khaetskii, D. Loss, L. Glazman, Phys. Rev. Lett. 88, 186802 (2002).

[12] W.A. Coish, D. Loss, Phys. Rev. B 70, 195340 (2004).

[13] W.G. van der Wiel et al., Rev. Mod. Phys. 75, 1 (2003).

[14] K. Ono, D.G. Austing, Y. Tokura, S. Tarucha, Science 297, 1313 (2002).

[15] A.C. Johnson, J.R. Petta, C.M. Marcus, M.P. Hanson, A.C. Gossard, cond-mat/0410679 (2004).

[16] The electrostatic gates are on the surface of a GaAs/AlGaAs heterostructure. The 2DEG is $90 \mathrm{~nm}$ below the surface, with density $1.33 \times 10^{11} \mathrm{~cm}^{-2}$ and mobility $9.71 \times 10^{5} \mathrm{~cm}^{2} / V s$. Measurements are performed in a dilution refrigerator at $150 \mathrm{mK}$, with a magnetic field in the plane of the heterostructure.

[17] Supporting text is available on Science Online.

[18] D. Paget, G. Lampel, B. Sapoval, V.I. Safarov, Phys. Rev. B 15, 5780 (1977).

[19] D. Gammon et al., Phys. Rev. Lett. 86, 5176 (2001).

[20] T. Fujisawa et al., Science 282, 932 (1998).

[21] O.N. Jouravlev and Y. Nazarov, private communications.

[22] The difference between the field dependence of the resonant and inelastic current can be explained by the coupling with the leads. The $\mathrm{S}(0,2)$ state is lifetime broadened due to coupling with the right lead $(\sim 0.3 \mu \mathrm{eV})$ giving a weaker field dependence for the resonant current. The 
field dependence of the inelastic leakage is not affected by the lead coupling, because under the high bias conditions of this experiment, there are no available states in the left lead that could broaden $\mathrm{S}(1,1)$.

[23] In the course of this experiment, we became aware of related measurements by A. C. Johnson et al., see Nature 435, 925 (2005).

[24] M. Dobers, K. v. Klitzing, J. Schneider, G. Weimann, K. Ploog, Phys. Rev. Lett. 61, 1650 (1988).

[25] A. K. Hüttel et al., Phys. Rev. B 69073302 (2004).

[26] The leakage current is sensitive to magnetic fields of only a few $\mathrm{mT}$, corresponding to $0.1 \%$ nuclear polarization. Given that the dot has $\sim 10^{6}$ nuclei, changes in the nuclear polarization of $0.1 \%$ can be caused by 1000 electron-nuclear flip-flop processes. For typical currents $(\sim 100$ fA) 1000 electrons move through the dot in one millisecond, so in principle current fluctuations as fast as $1 \mathrm{kHz}$ are possible.

[27] K. Ono, S. Tarucha, Phys. Rev. Lett. 92, 256803 (2004).

[28] R. de Sousa, S. Das Sarma, Phys. Rev. B 67, 033301 (2003).

[29] In Ref. 6], a $T_{2}^{*}$ of 16 ns was found in a GaAs quantum dot, slightly shorter than in our experiment, presumably due to a smaller dot size. A considerably shorter timescale, 500 ps, was measured in InAs dots [5], due to a notable difference in dot size, nuclear spin $I$, and the stronger hyperfine coupling constant in InAs.

[30] V.N. Golovach, A.V. Khaetskii, D. Loss, Phys. Rev. Lett. 93, 016601 (2004).

[31] J.M. Elzerman et al., Nature 430, 431 (2004).

[32] M. Kroutvar et al., Nature 432, 81 (2004).

[33] The authors are grateful to O.N. Jouravlev and Y. Nazarov for developing a model that helped greatly with the physical interpretation of the data, and to G. Burkard, W.A. Coish, V.N. Golovach, A.C.Johnson, D. Loss and C.M. Marcus for fruitful discussions. We thank R. Schouten, B. van den Enden and M. van Oossanen for technical assistance and J. Caro for supporting infrastructure. This work was supported by the DARPA-QUIST program, the Dutch Organisation for Fundamental Research on Matter (FOM), the Netherlands Organization for Scientific Research (NWO), the ONR, ERATO, and the EU-RTN network on spintronics. 

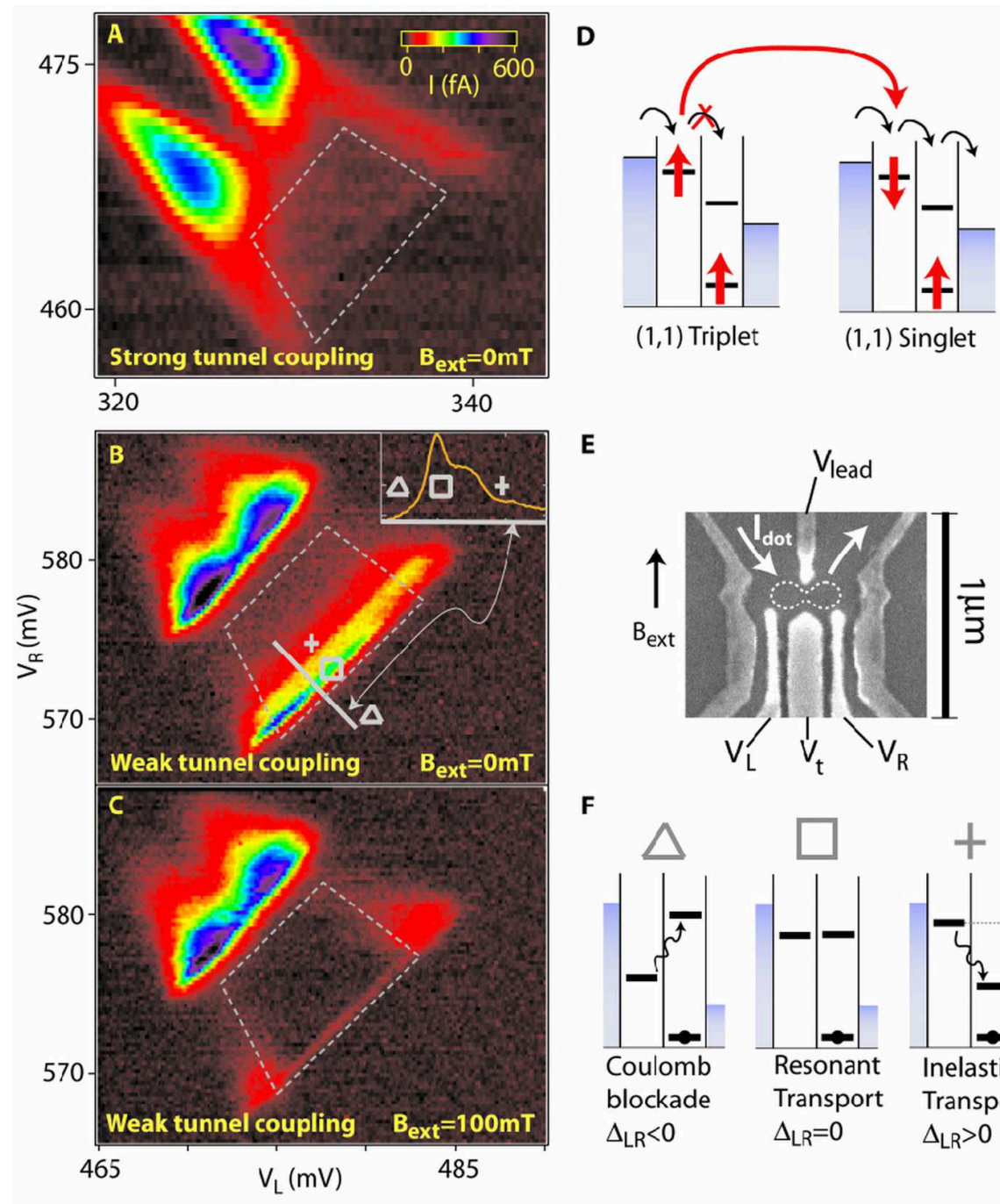

$\mathbf{E}$

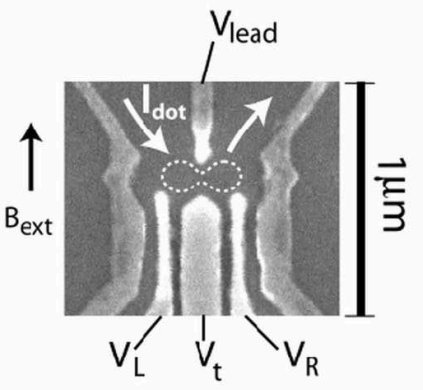

$\mathbf{F}$
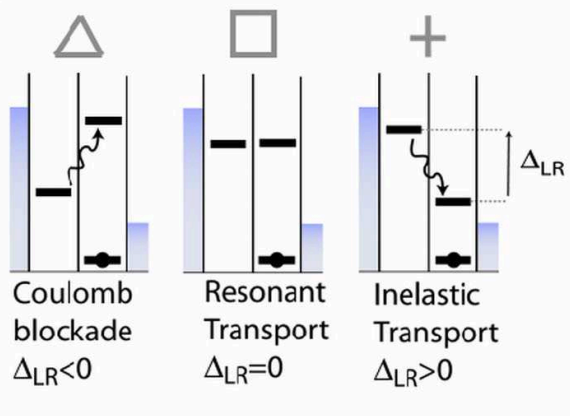

FIG. 1: Pauli blockade and leakage current. (A) Color-scale plot of the current through two coupled dots as a function of the left and right dot potentials (voltage bias $800 \mu \mathrm{eV}, V_{t}=-108$ $\mathrm{mV}$ ). The experimental signature of Pauli blockade is low current $(<80 \mathrm{fA})$ in the area denoted by dotted grey lines. (B) Analogous data for smaller interdot tunnel coupling $\left(V_{t}=-181 \mathrm{mV}\right)$, with the same color scale as in (A). A dramatic increase of leakage current is seen in the lower part of the Pauli blockaded area (green/yellow band). Inset: 1D trace along the solid grey line, with Coulomb blockaded, resonant and inelastic transport regimes marked, see also (F). (C) Analogous data for the same tunnel coupling as in (B), but for $B_{\text {ext }}=100 \mathrm{mT}$. The leakage current from (B) is strongly suppressed. (D) Two level diagrams that illustrate Pauli blockade in coupled quantum dots (see text). When the $(1,1)$ triplet is changed into the $(1,1)$ singlet (red arrow), Pauli blockade is lifted. (E) SEM micrograph showing the device geometry. White arrows indicate current flow through the two coupled dots (dotted line). (F) Level diagrams illustrating three transport regimes. $\triangle$ : Coulomb blockade; transport would require absorption of energy. $\square$ : Resonant transport; the dot levels are aligned. +: Inelastic transport; energy must be transferred to the environment, for instance by emitting a phonon. These symbols are used to denote inelastic, resonant, and Coulomb blockade regimes in Figs. 1, 2, and 3. 

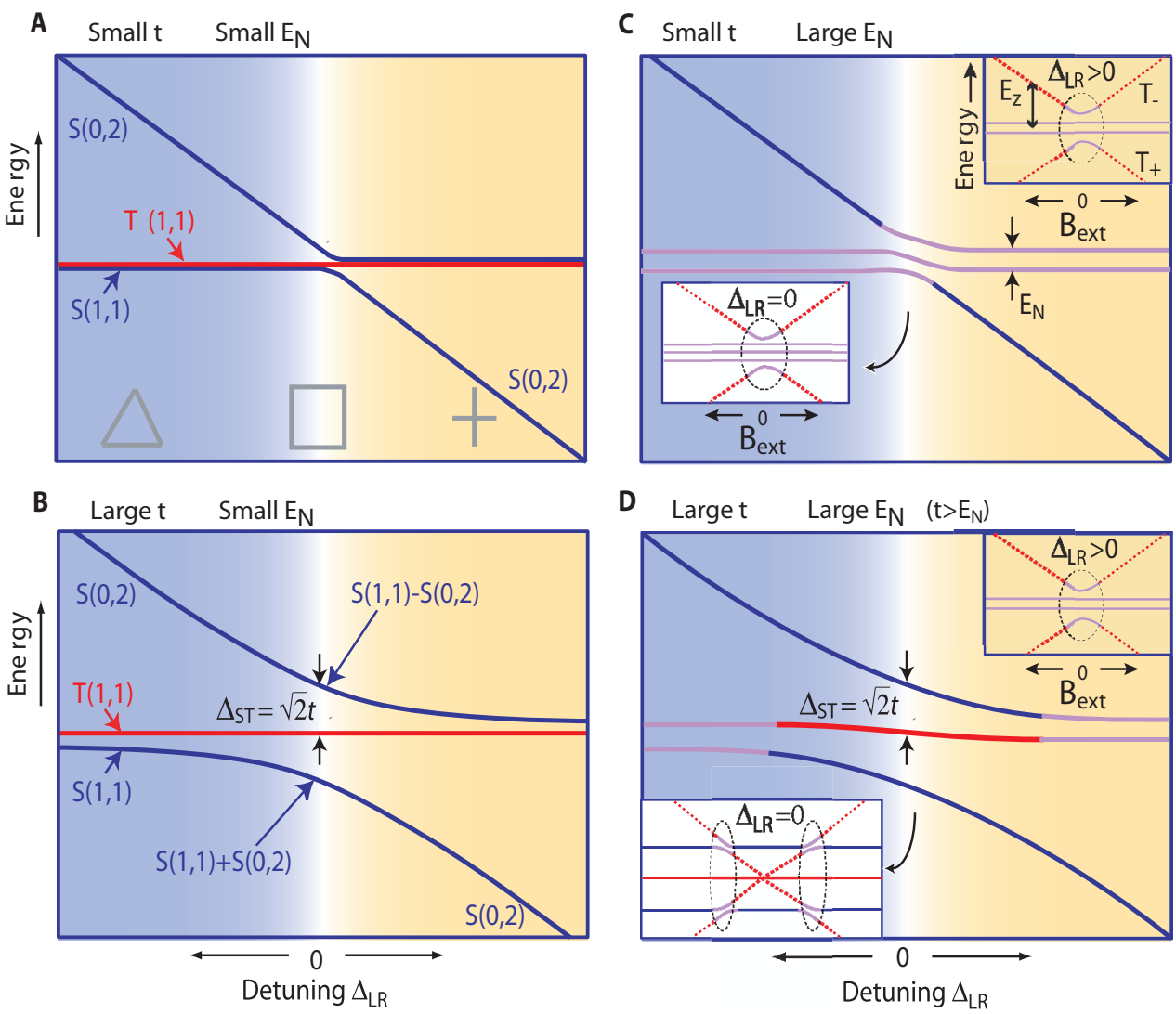

FIG. 2: Two-electron level diagrams showing energy as a function of detuning $\Delta_{L R}$. Detuning is defined so that the energy of $\mathrm{T}(1,1)$ remains constant as $\Delta_{L R}$ varies (see also Fig. S1B and the supporting online text). The panels on the left illustrate the effect of $t$; the panels on the right include the additional effect of an inhomogeneous magnetic field. Pure singlet and triplet states are drawn in blue and red respectively, strong admixtures in purple. The blue, white and yellow background correspond to the Coulomb blockade, resonant and inelastic transport regime respectively. (A) For small tunnel coupling, $\mathrm{T}(1,1)$ and $\mathrm{S}(1,1)$ are nearly degenerate. (B) For finite $t$, level repulsion between the singlet states results in a larger singlet-triplet splitting compared to (A), which depends on detuning. The tunnel coupling does not mix singlet and triplet states. For large $\Delta_{L R}$ (but smaller than the single dot S-T splitting) $E_{S T} \sim t^{2} / \Delta_{L R}$. (C and D) An inhomogeneous field mixes triplet and singlet states that are close in energy (purple lines). For clarity only one triplet state is shown in the main panels. (C) For small $t, \mathrm{~T}(1,1)$ and $\mathrm{S}(1,1)$ mix strongly over the full range of detuning. (D) For large $t, \mathrm{~T}(1,1)$ mixes only strongly with the singlet for large detuning. The insets to (C) and (D) show the effect of an external magnetic field on the two-electron energy levels. All three triplets are shown now; the triplets $\left|T_{+}\right\rangle$and $\left|T_{-}\right\rangle$split off from $\left|T_{0}\right\rangle$ due to $B_{\text {ext }}$. The leakage current is highest in the regions indicated by black dotted ellipses (see text). 

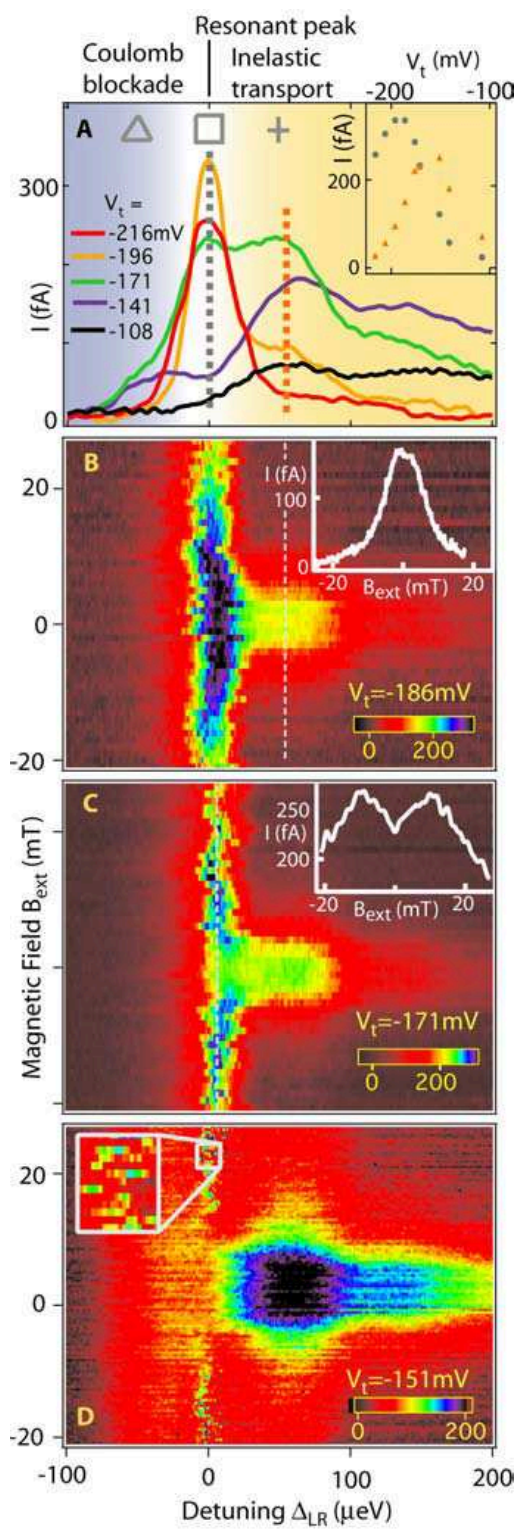

FIG. 3: The measured leakage current results from a competition between $E_{N}, E_{S T}$ and $E_{Z}$. (A) $1 \mathrm{D}$ traces of the leakage current as a function of detuning at $B_{\text {ext }}=0$, for a wide range of tunnel couplings (analogous to the inset of Fig. 1B). Coulomb blockade, resonant transport and inelastic transport are indicated by colored backgrounds as in Fig. 2. Inset: leakage current along the dotted grey and orange lines is shown as a function of $V_{t}$. Resonant and inelastic leakage (grey and orange markers) reach a maximum at different tunnel couplings $\left(V_{t}=-190 \mathrm{mV}\right.$ and -150 $\mathrm{mV}$ respectively). (B) For small tunnel coupling $\left(<E_{N}\right)$, both the resonant and inelastic leakage currents drop monotonically with $B_{\text {ext }}$. Inset: magnetic field dependence of the inelastic current along the dotted line $\left(\Delta_{L R}=40 \mu \mathrm{eV}\right)$. (C) For larger $t\left(>E_{N}\right)$, the resonant leakage current is maximum at $B_{\text {ext }} \pm 10 \mathrm{mT}$. Inset: field dependence of the resonant peak height (dotted line). (D) For still larger $t$, the resonant current is strongly reduced at low field, then becomes unstable for higher field (see inset). 

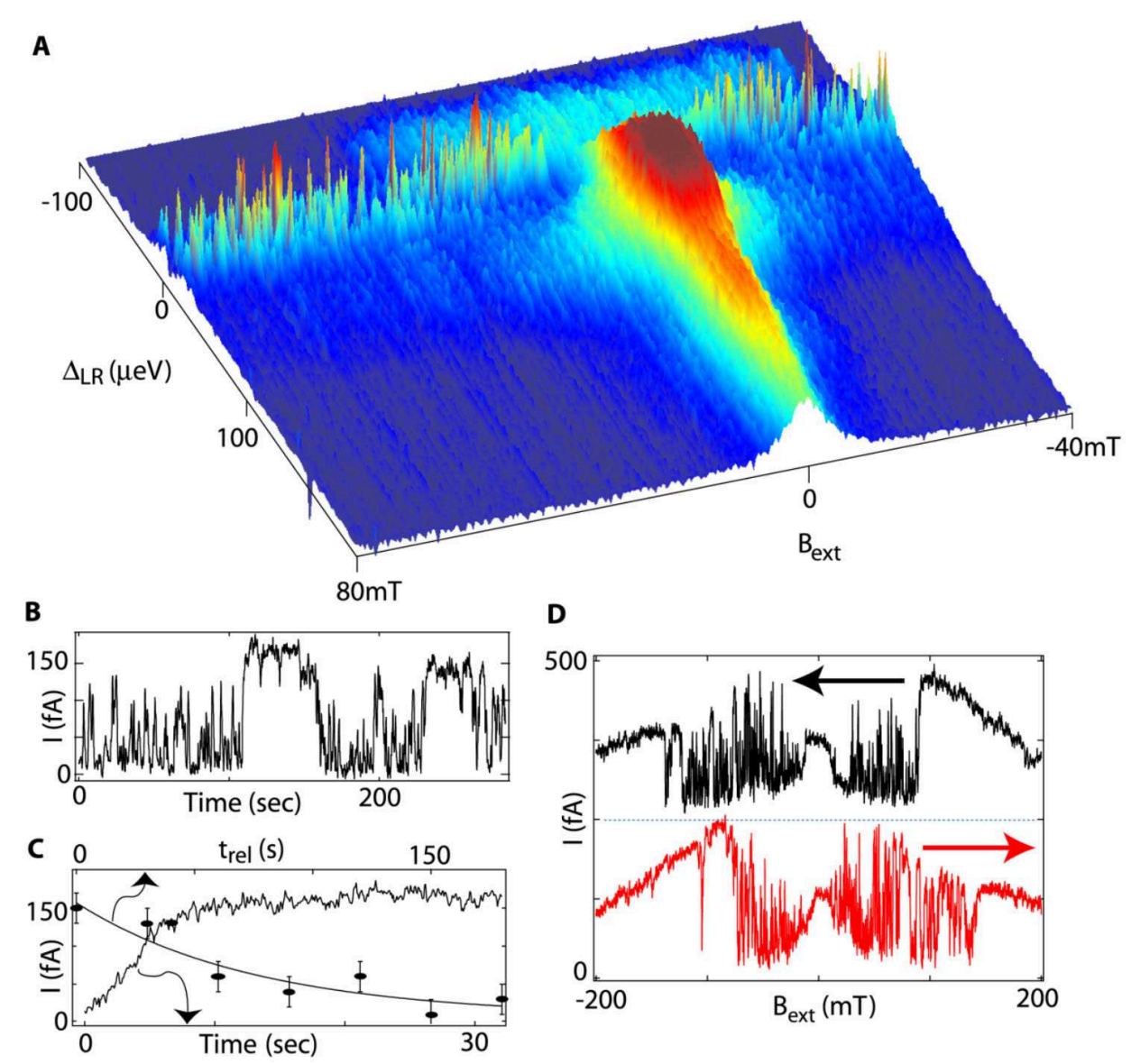

FIG. 4: Time dependence of the leakage current reveals the dynamics of the electron-nuclear system. This time dependence occurs in the regime corresponding to Fig. 2D. (A) Surface plot of electrical transport for $V_{t}=-151 \mathrm{mV}$. Instability on the resonant peak is visible as sharp current spikes. The sweep direction is from + to $-\Delta_{L R}$, for fields stepped from - to $+B_{\text {ext }}$. (B) Explicit time dependence of the resonant current exhibits bistability $\left(V_{t}=-141 \mathrm{mV}, B_{\text {ext }}=100 \mathrm{mT}\right)$. (C) Lower axis: dynamic nuclear polarization due to electron transport through the device $\left(V_{t}=-141\right.$ $\mathrm{mV}, \Delta_{L R}=0, B_{\text {ext }}=200 \mathrm{mT}$ ), after initialization to zero polarization by waiting for 5 minutes with no voltage applied. Top axis: in order to measure the nuclear spin relaxation time, we wait for the current to saturate, switch off the bias voltage for a time $t_{r e l}$, and then remeasure the leakage current. An exponential fit gives a time constant of $80 \pm 40 \mathrm{~s}$ (measurements of these long timescales result in large error bars, $\pm 20 \mathrm{fA}$ due to $1 / f$ noise). (D) The field dependence of the resonant current is hysteretic in sweep direction $\left(V_{t}=-149 \mathrm{mV}\right)$. Each trace takes $\sim 7$ minutes. 